\title{
Políticas de transporte público en países en desarrollo; transición a la megaciudad moderna
}

Manuel José Martinez Espinal ${ }^{*}$

\section{INTRODUCCIÓN}

En este texto se proponen políticas de transporte público para una nueva situación. Si la megaciudad tradicional era intensiva en el uso del transporte público, y era muy congestionada; la megaciudad moderna es ahora menos intensiva en el uso del transporte público, hace más frecuente uso de taxis y autos privados, y es todavía más congestionada. Estos cambios se deben al rápido proceso de motorización reciente (compra masiva de autos privados). En la megaciudad tradicional se aconsejaba la reducción del número de buses de transporte público para combatir la congestión. Sin embargo, nuevas mediciones han descubierto que un alto número de vehículos de transporte público no causa una congestión tan grave como la que se suponía, y que la reducción de buses de transporte público resultaría imperceptible para bajar la congestión. Por lo tanto, se necesitan nuevas políticas en el campo del transporte público como también en el campo de la congestión. Justamente, este artículo presenta nuevas políticas para megaciudades modernas en su proceso de transición. Un índice de capacidad evitará el desvío de fondos públicos a proyectos que reduzcan la capacidad o la productividad de transporte. Un índice de pasajero asegurará que el servicio de transporte público mejore cuando se usen fondos públicos. El transbordo desde el transporte público rápido masivo al transporte público de buses debe cumplir con minimizar la distancia de caminata hasta un límite de 30 metros. Así también, se propone la condición de fluctuación del valor del pasaje a la entrada de nuevas unidades al mercado del transporte público. Finalmente, las intervenciones gubernamentales sobre las vías saturadas deben asegurar equidad sobre la población.

\footnotetext{
* Consultor y profesional independiente. Calle Schrader 179, dpto. 202, San Borja, Lima 41, Perú. Tel: 011-511-22-44-028; email: mjmartinez@pucp.pe
} 
Lima y Callao es una megaciudad moderna de 9,5 millones de habitantes (INEI, 2019), cuya productividad es baja y cuyos salarios ascienden a menos del $9 \%$ que los de un país desarrollado. Transporte es uno de sus sectores con mayor cantidad de trabajadores.

La demanda de transporte motorizado era de 12,1 millones de viajes diarios en el 2005, un $77 \%$ de los cuales usaba transporte público de buses (TPB). Esta demanda crecerá hasta 18 millones en 2025, cuando un 71\% usará TPB (Agencia de Cooperación Internacional del Japón [JICA], 2005). Mientras que la demanda de TPB crecerá $37 \%$, la de taxis y autos privados crecerá $93 \%$, causando graves problemas de congestión, a menos que se invierta en transporte público rápido masivo (TPRM), en mejoras al TPB, y en vías expresas.

En el contexto de este artículo, Lima y Callao sirve para entender a otras megaciudades en su camino a la modernidad a causa de sus niveles extremos de capacidad vial y de tamaño de flota. Por un lado, su capacidad vial es muy pequeña, y por el otro lado, el número de buses de transporte público es muy grande. Solamente hay una vía expresa radial de tres carriles por sentido por unos $10 \mathrm{~km}$, dedicada al uso de taxis y autos privados, y una sola carretera diametral de tres o cuatro carriles por sentido de unos $48 \mathrm{~km}$, dedicada al tráfico mixto. La flota de TPB es de unos 40000 vehículos-hora registrado en 2004, con buses de diverso tamaño y distribuido en 600 rutas (JICA, 2005).

Paralelamente, el proceso de motorización y el crecimiento de la demanda de transporte privado reducirá la velocidad promedio a la mitad, en horas-pico, desde $16,8 \mathrm{~km} / \mathrm{h}$ a 7,5 km/h sobre el período 2005-2025 (JICA, 2005). Desde entonces, el número de buses TPB se ha reducido debido a las políticas recomendadas tradicionalmente para combatir la congestión.

Las autoridades han implementado las políticas convencionales aconsejadas para TPB (Martínez, 2007); la desregulación se realizó en 1991 y tuvo éxito en mejorar el servicio. Más tarde, los intentos de re-regulación iniciada entre 2001 y 2004 trató de combatir la congestión reduciendo drásticamente el número de vehículos de TPB. Sin embargo, recientes mediciones (Martínez, 2017), han descubierto que la congestión no se vería mayormente afectada por la reducción del número de vehículos del TPB.

Más allá de su efecto sobre la congestión, al reducir la flota de buses, las autoridades colocan en riesgo de colapso al TPB al mejorar la posición competitiva del transporte de taxis y autos privados y empujar la demanda hacia el transporte privado. Este peligro lleva a la necesidad de nuevas políticas que sean efectivas en mejorar el TPB de Lima y Callao en su competitividad sobre el transporte de taxis y autos privados.

Antes de entrar en la sección del supuesto básico, es importante describir la megaciudad y de cómo su forma y su crecimiento afectan al diseńo de los TPRM. Hasta la década de 1960 el TPB permitió construir en los alrededores de las líneas de tranvías. Desde la misma década, las rutas de buses permitieron expandir asentamientos en los espacios existentes entre las líneas de tranvías, vale decir entre la línea de Lima-Chorrillos al sur, la de Lima-Magdalena al sur-oeste y Lima-Callao al oeste, conformando el triángulo Lima-Chorrillos-Callao, con un corredor central Lima-Miraflores intenso en tráfico. 
También las rutas de TPB que corrían sobre las carreteras salientes desde Lima—al norte, al centro y al sur-permitieron extender la ciudad en "conos». En estos conos predominan sectores de más bajos ingresos aprovechando terrenos de menor valor. En consecuencia, Lima y Callao es una ciudad que ha crecido y se ha formado de forma compacta, gracias al transporte TPB, permitiendo una gran expansión. Una característica resultante es la alta cantidad de transbordos entre rutas de buses.

Recientemente, se han efectuado ingentes inversiones públicas en TPRM, consiguiéndose construir y operar un sistema de buses rápidos metropolitano de $26 \mathrm{~km}$ que sirve un millón de viajes diarios, y un tren eléctrico de $35 \mathrm{~km}$ que sirve 320 mil viajes diarios. La alta tasa de transbordos natural del sistema TPB se intensifica en los nuevos TPRM y por ello tienen una necesidad especial por integración física que merece una política adicional para estos transbordos que regule el diseño de las estaciones de TPRM.

\section{REVISIÓN DEL SUPUESTO BÁSICO Y SUS CONSECUENCIAS}

El soporte crucial de la re-regulación del TPB ha sido que la desregulación había llevado a un desproporcionadamente alto número de pequeños buses de transporte público, causando una sobreoferta que producía una congestión innecesariamente grave. La libre competencia era insostenible porque producía externalidades de costo gravoso que son la congestión y la contaminación. Las autoridades municipales debían reducir el número de vehículos de transporte público hasta un mínimo.

Este supuesto básico ha sido revisado para Lima y Callao (Martínez, 2017) y se ha encontrado que ya no se cumple. Durante la década de 1990, la desregulación permitió la libre entrada legal de operadores privados en desproporción, lo que produjo gran congestión y contaminación (Gwilliam y Scurfeld, 1996). Se recomendó re-regular para reducir el número de vehículos del TPB, por medio de concursos públicos, porque había una sobreoferta que podía ser eliminada (Estache y Gómez-Lobo, 2004). Congestión y contaminación eran fallas del libre mercado, que imponían límites de factibilidad a la libre competencia y que por ello era necesaria la re-regulación. Incluso, en el trabajo de Ardila (2008) se determinaba que la sobreoferta era el problema a resolver, porque la congestión mínima se conseguiría luego que la sobreoferta era eliminada. Sin embargo, nunca se midió la congestión, dándola por supuesta. Cuando esta congestión se midió (Martínez, 2017) se encontró sorprendentes resultados que se exponen a continuación.

Los cambios de velocidad fueron estimados usando un modelo de transporte en software Dynasmart midiendo los efectos de flujos de buses. Los datos son del Plan Maestro 2005-2025 (JICA, 2005) usados en un proyecto financiado por el Consejo Nacional de Ciencia y Tecnología del gobierno peruano (Concytec) mediante contrato 194-2005-CONCYTEC/OAJ. Los resultados son los siguientes:

- La velocidad de la ciudad cae en $-76 \%$ en tres horas (4 a 7 a.m.) debido a la demanda de taxis y autos privados. 
- La velocidad se reduce adicionalmente en -37\% a causa del crecimiento 2005-2025 de la demanda de taxis y autos privados.

- En cambio, si el TPB redujera su flota en la mitad, la velocidad se incrementaría en:

- $7 \%$ para toda la ciudad.

- 3,5\% considerando solo el objetivo de 2014 sobre corredores selectos.

- 2,4\% si buses estándares (más grandes) se usaran en 2014.

- $9 \%$ pero a las 11 am cuando es menos necesario.

- Además, los casos graves de congestión causada por el TPB se limitan a:

o Cortos períodos de 4 a 15 minutos, durante las horas-pico de 7 a.m., 1 p.m. y 6 p.m.

- Pocas zonas de la ciudad: cerca de los centros de Lima, San Isidro y Miraflores, y donde la capacidad vial es insuficiente.

En reducción de emisiones contaminantes, se encontró que reducir la flota del TPB a la mitad no sería la mejor alternativa (Martínez, 2017) pues su efecto sería similar al obtenido por Inspección y Mantenimiento. Pero, de cambiar todos los taxis a combustible gas se obtendría el doble de efectividad o, de cambiar todos los buses a gas se obtendría ocho veces de efectividad.

No solamente el supuesto básico no se cumple, sino que su aplicación ha llevado a problemas más graves aún, tales como hacer perder competitividad al TPB empujando la demanda hacia taxis y autos privados, y a cometer políticas inequitativas, todo con medidas gubernamentales financiadas con fondos públicos, lo cual se analiza a continuación.

El primer corredor que se implementó fue el Corredor Azul en las vías que conectan el viejo centro de Lima con el nuevo centro de Miraflores. La capacidad se redujo a un mínimo, causando que el servicio a los pasajeros se desmejore en más de un aspecto, a pesar de las mejoras obtenidas con vehículos más grandes y con mayor velocidad. La capacidad productiva fue reducida desde 750 vehículos por hora con una capacidad promedio de 33 pasajeros (datos de JICA [2005] que mencionan un flujo de 500-1000 buses por hora, 714 en algún punto), hasta 150 vehículos por hora con una capacidad de 80 pasajeros; una reducción de capacidad de $-52 \%$. Las rutas alimentadoras fueron también reducidas, y el espaciamiento de paraderos se alargó hasta un mínimo de 400 metros en vez de los 100 metros usuales, aún en las zonas céntricas. Entonces, las pérdidas en los tiempos de caminata y de espera, contrabalancearon las aparentes ganancias de tiempo de viaje en el vehículo. Ocurrieron protestas y algo de violencia y el descontento popular fue reportado por los medios de comunicación por varias semanas hasta que la demanda se dirigió naturalmente hacia otros corredores. Entonces, no solamente se redujo la capacidad, sino que también se desmejoró el servicio (Martínez, 2017). Los líderes del proceso de re-regulación explicaron el descontento público como el costo político necesario para mejorar el TPB (Rojas García, 2017). De repetirse esta constante 
desmejora del TPB en varios corredores, y supuestamente obtenerse menor congestión, entonces la intervención gubernamental desmejorará la productividad y la competitividad del TPB en favor de taxis y autos privados.

Las autoridades municipales aplicaron la política recomendada mediante la ley de vías saturadas; cuando una vía está saturada, las autoridades pueden intervenirla. En 2014 todas las vías con TPB fueron declaradas saturadas, y se intervino únicamente el TPB, en vez de intervenir equitativamente incluyendo a taxis y autos privados. Las autoridades cancelaron las autorizaciones de rutas TPB. Era inequitativo porque la mayoría de pasajeros en TPB son más pobres, aunque era una medida rápida para reducir el número de buses de TPB con nuevos concursos públicos (Martínez, 2017). Sin embargo, las intervenciones gubernamentales tienen la obligación de mantener la equidad sobre la población, de violarse este principio, se producen injusticias sociales con costos a financiarse o corregirse.

\section{LA NUEVA SITUACIÓN}

Las megaciudades han llegado a una nueva situación. Ya no es la década de 1980 de la escasez del número de buses que necesitaba desregulación, ni tampoco los 2000 de la abundancia de buses que necesitaba re-regulación, sino que es una nueva situación de congestión insoportable causada por el transporte de taxis y autos privados en medio de un proceso rápido de motorización de la megaciudad, y es cuando una gran inversión pública se necesita con mayor urgencia en transporte urbano.

Sin embargo, las actuales políticas han reducido la capacidad del TPB y han desmejorado su servicio a los pasajeros, y por ello, la demanda de transporte está dejando atrás al TPB y adoptando taxis y autos privados, a una tasa muy alta y comparable a los procesos que ya han sucedido antes en los países desarrollados. Un proceso artificialmente acelerado de motorización puede reducir al TPB a una actividad pequeña o insolvente, tal que, en una situación de baja productividad, y sin que la población pueda mudarse, pueda llegarse a una congestión exacerbada que malogre la competitividad y el crecimiento económico de la megaciudad. Nuevas políticas deben proponerse con el objetivo de evitar la posibilidad de colapso del TPB.

La primera política es que la capacidad de TPB debe protegerse y al mismo tiempo asegurar que se mejore el servicio al pasajero del TPB. ¿Cómo hacerlo? En el contexto peruano el sistema nacional de inversión pública ha sido simplificado al mínimo desde el 2017 con el objetivo de agilizar el sector gubernamental (MEF, 2017). Por ello, nuevos Índices deben desarrollarse de forma simple, no-burocrática, para alcanzar estos objetivos de una manera expeditiva: un índice de capacidad (IC) para calcular los incrementos en la capacidad de transporte, y un índice de pasajero (IP) para calcular las mejoras en el servicio del transporte.

La segunda política es que la accesibilidad medida como distancia de caminata entre los sistemas de TPRM y las rutas de TPB debe regularse. Hasta ahora no se ha publicado 
que los TPRM en países en desarrollo están fallando en minimizar la distancia de caminata para transbordos a TPB. En Lima y Callao, las existentes estaciones de TPRM se han construido demasiado lejos de los paraderos de las rutas TPB que pasan por las avenidas perpendiculares, haciendo muy difícil el transbordo entre los dos sistemas. En promedio, el pasajero tiene que caminar 200 metros desde la estación de tren eléctrico hasta el paradero, y 100 metros desde la estación del Metropolitano de Buses Rápidos.

La tercera política se refiere al valor del pasaje. Desde los inicios de la década de 1990 el pasaje ha quedado estable a S/.1,00. Pero los nuevos corredores re-regulados fijaron su pasaje en S/.1,70 al implementarse desde 2014. Sin embargo, los nuevos corredores con nuevos vehículos más grandes han tenido déficits operativos, como han informado a través de los medios de prensa. Luego de la experiencia del Corredor Azul, la conveniencia de los subsidios para el nuevo TPB ha sido propagado por políticos (Rojas García, 2017) y por académicos (Alegre-Escorza y Alarcón, 2016) como soluciones políticas (sea porque los pobres necesitan un pasaje más económico, o porque el TPB es un servicio público, o porque el nuevo sistema tronco-alimentador genera transbordos adicionales que hacen más caro al TPB). El hecho es que, luego de implementarse el nuevo valor del pasaje del Corredor Azul las autoridades dijeron que la demanda se había desplomado desde 260000 pasajeros diarios a solamente 160 000, una caída de -38\%. Por eso se necesitaban subsidios. Podría decirse que los nuevos sistemas que operaron al nivel de los estándares más modernos eran insostenibles a los niveles de productividad de la población urbana de 2014, porque el ingreso personal de los pasajeros no era suficiente. Así pues, una nueva regulación debe hacerse, para evitar los subsidios o moderarlos, y posiblemente permitir al TPB operar a un nivel de productividad que pueda estar al alcance de los salarios de la población. De esta manera, el TPB será sostenible. Esto significa, básicamente, permitir a los sistemas TPB tradicionales y modernos, poner un precio del pasaje sostenible y operar con capacidad suficiente sin cerrar la entrada a nuevos vehículos. Esta política permite a los sistemas TPB operar con capacidad suficiente y alcanzar el equilibrio en el mercado, por medio de una simple regulación basada en una combinación de valor flotante del pasaje con mejoras en el servicio TPB.

La cuarta política se refiere a la manera de combatir la congestión de una manera equitativa. Esto significa diseñar una fórmula para dosificar equitativamente los vehículos de TPB y de taxis y autos privados, usando el costo generalizado sobre las arterias saturadas. En la siguiente sección se presenta las nuevas políticas en detalle.

\section{LAS NUEVAS POLÍTICAS}

\section{El índice de capacidad IC}

El índice de capacidad IC es un índice simple a ser calculado antes de que la inversión pública se ejecute. El IC consiste en la siguiente fórmula: 


$$
I C=\frac{C_{2} * S_{2}}{C_{1} * S_{1}}
$$

donde:

$I C=$ índice de capacidad

$1,2=$ situación inicial, situación final

$C=$ capacidad en pasajeros por hora por sentido

$S=$ velocidad comercial

Cuando $I C \geq 1$, está bien continuar con la inversión, si no, entonces, adicionar más capacidad en la situación final. Este índice asegura que la capacidad productiva no se reduzca a causa de la intervención gubernamental en esta vía de transporte.

Por ejemplo, en el Corredor Azul, se tiene que $C_{1}=750 \times 33=24750$ (consistentes con conteos hechos en JICA (2005) los cuales registraron 20000 a 38000 pasajeros por hora por dirección en este segmento), $S_{1}=9 \mathrm{~km} / \mathrm{h}, C_{2}=150 \times 80=12000, S_{2}=$ $14 \mathrm{~km} / \mathrm{h}$, el resultado es $C I=0,75$; por lo tanto se requiere ańadir más capacidad a la situación final.

\section{Índice de pasajero IP}

El índice de pasajero IP se basa sobre el costo generalizado aplicado al pasajero promedio. Consiste en la siguiente formula:

$$
I P=\Delta e n-\text { vehi }_{t} * V_{\text {en-vehi-t }}+\Delta \text { espera }_{t} * V_{\text {espera-t }}+\Delta \text { caminar }_{t} * V_{\text {caminar }-t}+\Delta \text { pasaje }
$$

donde:

$I P=$ índice de pasajero

$D=$ cambio de la situación final menos la situación inicial

en-veh $h_{t}=$ tiempo gastado a bordo del vehículo, en minutos

espera $a_{t}=$ tiempo gastado en esperar, en minutos

caminar $_{t}=$ tiempo gastado en caminar, en minutos

pasaje $=$ valor de un pasaje, en centavos

$V=$ valor del tiempo en centavos por minuto

Cuando el IP [ 0 , está bien el proyecto porque baja, si no, entonces debe incrementarse la frecuencia, o bajar el valor del pasaje para restaurar la satisfacción del pasajero. Este índice asegura que el servicio no se desmejore usando fondos públicos especialmente cuando uno de sus atributos se mejora cuando los demás se desmejoran.

Por ejemplo, en el Corredor Azul, $\Delta$ en-veh $i_{t}=-12^{\prime}, \Delta$ sesper $a_{t}=+2^{\prime}, \Delta$ caminar $_{t}=$ $+150 \mathrm{~m} / 80 \mathrm{~m} / \mathrm{min}=+1,9^{\prime}$, ppasaje $=+70+100=+170 \phi$ (se añade el transbordo); los valores del tiempo son $V_{\text {en-veh-t }}=1,7 \$ / \mathrm{min}, V_{\text {espera- } t}=7,3 థ / \mathrm{min}, V_{\text {caminar }-t}=7,6 \Phi / \mathrm{min}$, tomados de (Martínez y Cornejo, 2003). El resultado es $-20+15+15+170=180$ céntimos de más. Esto indica que la nueva situación es peor que la anterior porque 
el costo generalizado promedio es más alto. Por ello, se recomienda bajar los pasajes y/o mejorar el servicio en otros atributos antes de implementar el proyecto o la intervención gubernamental.

\section{Distancia mínima de caminata para transbordos de TPRM}

Se ha encontrado que las estaciones de TPRM están demasiado lejos de los paraderos de TPB de las calles perpendiculares. Estos valores afectarán considerablemente la posición competitiva de los sistemas TPRM a pesar de la cuantiosa inversión pública que han costado. Por ejemplo, sea que el pasajero promedio de la Línea 1 del tren eléctrico está caminando cerca de 180 metros de más, pues esto significaría unos 2'30" más de caminata, si se toma en consideración el valor del tiempo de caminata de 7,6 $\$ / m i n$ mientras que el valor del tiempo en el vehículo es $1,7 \$ / \mathrm{min}$. Con el fin de compensar por este mayor costo, el viaje en el tren eléctrico se tendría que reducir en $2^{\prime} 30^{\prime \prime *}(7,6 / 1,7)=$ $11^{\prime}$, lo cual es imposible en una línea que ya está operando a una velocidad comercial de $38 \mathrm{~km} / \mathrm{h}$. Estos costos se deben cortar regulando que la distancia de caminata sea de 30 metros o menos, desde la puerta de la estación del TPRM hasta el paradero de TPB de la vía perpendicular, dada la importancia de los transbordos en los sistemas de Lima y Callao.

\section{Precio fluctuante del pasaje y regulación de la entrada al mercado}

El precio del pasaje en el tradicional TPB se mantuvo en $S / .1,00$ y no se necesitaron subsidios, mientras que el pasaje del re-regulado TPB se colocó en $S / .1,70+1,00=$ 2,70 y se necesitaron subsidios los cuales se han pedido públicamente de varias maneras. Los subsidios propuestos de esta manera son innecesarios si fuera posible mantener e incrementar la productividad del nuevo TPRM a un nivel más elevado, pero a un costo que pueda pagar la población, teniendo en cuenta sus bajos ingresos propios de un país en desarrollo. Por lo tanto, debe regularse que en vez de incurrir en la necesidad de subsidios, el nuevo TPB debe ser elevado a una productividad más alta de tal manera que sus costos operativos y ganancias sean cubiertos por el precio del pasaje del mercado. El precio del pasaje debe fluctuar y, cuando suba reflejando la escasez de vehículos de TPB permita la entrada de vehículos hasta que la productividad sea tal que pueda ser pagada por la población.

\section{Dosificación equitativa de vías saturadas}

Hasta recientemente, la ley de vías saturadas ha incidido solo sobre el TPB. Se necesita una formula más equitativa. Aquí se pone en manos de las autoridades un instrumento simple de dosificación equitativa que se logra utilizando los valores relativos del tiempo en vehículo de los pasajeros del TPB $(1,7 \$ / \mathrm{min})$ y el de los de vehículos privados (8,9 \$/min) (Martínez y Cornejo, 2003). 
Por ejemplo, una céntrica avenida en una dirección puede acomodar 600 autos por hora transportando 2 pasajeros y 400 vehículos TPB transportando 20 pasajeros (AATE, 2002). Entonces, el costo total por minuto es de $600 \times 2 \times 8.9460+400 \times 20 \times 1.7460=$ $405 \%$, distribuido en $45 \%$ costo de autos y 55\% costo de TPB. Si los vehículos TPB son de un tamaño equivalente de 2 autos privados, entonces la congestión total es causada por $600+400 \times 2=1400$ auto-equivalentes. Si se erradica la congestión con una reducción del flujo a unos 1000, entonces 400 auto-equivalentes necesitan ser sacados a otra ruta por medio de una dosificación. La proporción reparte los 400 en 180 autos (45\%) y 220/2 = 110 vehículos TPB (55\%). Al final la dosificación será 1 auto privado sacado de la vía y 2 permitidos que circulen por la vía, y 1 vehículo TPB sacado de la vía y 3 permitidos que circulen por la vía; o, si se permite una cola de espera, pueden entrar 2 autos privados y 3 vehículos de TPB por cada medio minuto. Se dosifica el tráfico de manera equitativa para combatir la congestión durante las horas-pico y en las zonas céntricas o en las zonas de escasa capacidad vial. Sería simple tomar conteos de vehículos por 15' y luego introducir los datos en una aplicación de un teléfono celular que asista al oficial de policía a cargo de la vía saturada. Puede luego incluirse en el sistema semafórico computarizado fácilmente si se segrega por tipo de vehículo.

\section{CONCLUSIONES Y RECOMENDACIONES}

En primer lugar, el supuesto de la re-regulación del TPB en países en desarrollo ya no se cumple. En vez de combatir a la congestión, la reducción de vehículos de TPB ha bajado su capacidad y desmejorado el servicio. Como resultado, se ha exacerbado el proceso de motorización acelerándolo, poniendo en riesgo la factibilidad del TPB. Esta nueva situación ha sido creada en una megaciudad moderna con poca capacidad vial y alto número de vehículos de TPB, y estas conclusiones pueden ser utilizadas en megaciudades similares.

En segundo lugar, a medida que las megaciudades crecieron sobre la base del TPB, los transbordos entre rutas TPB se han vuelto muy importantes, y se intensifica esta importancia en las estaciones TPRM. Por ello, es necesario reducir los costos de caminata innecesaria con un límite máximo de 30 metros desde la estación TPRM hasta el paradero TPB de la vía perpendicular.

En tercer lugar, en este artículo se han propuesto nuevas políticas, que son simples, ágiles y rápidas de ser implementadas. Un índice de capacidad evitará reducir la capacidad de transporte gastando dineros públicos. Un índice de pasajero asegurará que el servicio mejore como resultado del gasto público. El valor del pasaje debe fluctuar libremente para que al subir, permita ver la señal para que se incremente el número de vehículos de TPB que optimice el servicio a un nivel de productividad que la población pueda costear, sin subsidios innecesarios, cuando esto sea posible. Finalmente, se presenta un instrumento de dosificación equitativa de tráfico para intervenir vías saturadas. 


\section{REFERENCIAS}

Agencia de Cooperación Internacional de Japón - JICA (2005). Plan Maestro de Transporte Urbano para el Área Metropolitana de Lima y Callao. Lima: JICA.

Alegre-Escorza, Mariana y Gonzalo Alarcón Rodríguez-Paiva (2016). Transporte urbano: ¿Cómo resolver la movilidad en Lima y Callao? Lima: Consorcio de Investigación Económica y Social - CIES. Recuperado de www.cies.gob.pe/sites/default/files/investigaciones/dp_ transporte_urbano_sep.pdf

Ardila, Arturo (2008). Limitation of Competition in and for the Public Transportation Market in Developing Countries. Lessons from Latin American Cities. Transportation Research Record: Journal of the Transportation Research Board, 2048, 8-15. Washington, D.C.: Transportation Research Board of the National Academies.

Autoridad Autónoma del Tren Eléctrico - AATE (2002). Circulación de Buses Tomo II; Análisis de Capacidady Niveles de Servicio de las Intersecciones de las Lineas Metro-Bus; Diseño de Buses Rápidos. Reporte de Trabajo. Lima: AATE.

Estache, Antonio y Andres Gomez-Lobo (2004). The Limits of Competition in Urban Bus Services in Developing Countries. World Bank Policy Research Working Paper 3207. https://doi. org/10.1596/1813-9450-3207

Gwilliam, K. y R. Scurfeld (1996). Competition in Public Road Passenger Transport. Paper TWU39. Washington, D.C.: The World Bank.

INEI (2018). Censos Nacionales 2017: XII de Población y VII de Vivienda; Perú: Crecimiento y Distribución de la Población, 2017; Primeros Resultados. Lima: Instituto Nacional de Estadística e Informática. Recuperado de https://www.inei.gob.pe/media/MenuRecursivo/ publicaciones_digitales/Est/Lib1530/libro.pdf

Martínez, Manuel J. (2007). Los desafíos confrontados por los proyectos convencionales de transporte y el potencial de los sistemas inteligentes de transporte para una ciudad en desarrollo, Lima, Perú. ECIPERU Revista del Encuentro Científico Internacional, 4(1), 14-17. Recuperado de https://revistaeciperu.files.wordpress.com/2016/07/eci2007vvol4num1.pdf

Martinez, Manuel J. y Javier Cornejo (2003). Value of the Facilities and Attributes of New Heavy Rail and Bus Rapid Transit Projects in a Developing City. The Case of Lima, Peru. Transportation Research Record: The Journal of the Transportation Research Board 1835, 50-58. Washington, D.C.: TRB of the National Academies.

Martínez, Manuel José (2017). Transporte público de buses versus congestión y contaminación en Lima y Callao. Economía, 49(79), 47-86. https://doi.org/10.18800/ economia.201701.002

Ministerio de Economía y Finanzas - MEF, Perú (2017). Reglamento del Decreto Legislativo 1252 que crea el Sistema Nacional de Programación Multianual y Gestión de Inversiones. Lima-Peru. Recuperado de http://www.mef.gob.pe/es/normatividad-inv-publica/instrumento//decretos-supremos/15604-decreto-supremo-n-027-2017-ef-1/file

Rojas García, Gonzalo Alonso (2017). La coordinación en el proceso de implementación de la reforma del transporte en Lima Metropolitana: el caso del Corredor Azul. Tesis para optar el título de licenciado en Ciencia Política y Gobierno. PUCP, Lima-Perú. Recuperado de http://tesis.pucp.edu.pe/repositorio/handle/20.500.12404/10152

Documento recibido el 19 de octubre de 2018 y aprobado el 20 de diciembre de 2019 